\title{
Characterization of the Stereoselective P450 Enzyme BotCYP Enables the In Vitro Biosynthesis of the Bottromycin Core Scaffold
}

\author{
Sebastian Adam, ${ }^{+, \neq}$Laura Franz, ${ }^{+, \neq}$Mohammed Milhim, ${ }^{\S}$ Rita Bernhardt, ${ }^{\S}$ Olga V. Kalinina ${ }^{\circ}$ and Jesko Koehnke ${ }^{+,,,^{*}}$
}

+Workgroup Structural Biology of Biosynthetic Enzymes, Helmholtz Institute for Pharmaceutical Research Saarland (HIPS), Helmholtz Centre for Infection Research (HZI), Saarland University, Campus Geb. E8.1, 66123 Saarbrücken, Germany

${ }^{\S}$ Department of Biochemistry, Saarland University, Campus Geb. B2.2, 66123 Saarbrücken, Germany

${ }^{\circ}$ Prof. Dr. Olga V. Kalinina, Drug Bioinformatics Group, HIPS, HZI, Saarbrücken, Germany. Medical Faculty, Saarland University, Homburg, Germany

School of Chemistry, University of Glasgow, Glasgow, UK. Email: Jesko.koehnke@glasgow.ac.uk

Supporting Information Placeholder

\begin{abstract}
Bottromycins are ribosomally synthesized and posttranslationally modified peptide natural product antibiotics that are effective against high-priority human pathogens such as methicillinresistant Staphylococcus aureus. The total synthesis of bottromycins involves at least 17 steps with a poor overall yield. Here, we report the characterization of the cytochrome P450 enzyme BotCYP from a bottromycin biosynthetic gene cluster. We determined the structure of a close BotCYP homolog, and used our data to conduct the first large-scale survey of P450 enzymes associated with RiPP biosynthetic gene clusters. We demonstrate that BotCYP converts a Cterminal thiazoline to a thiazole via an oxidative decarboxylation reaction and provides stereochemical resolution for the pathway. Our data enable the 2-pot in vitro production of the bottromycin core scaffold and may allow the rapid generation of bottromycin analogues for compound development.
\end{abstract}

Ribosomally synthesized and post-translationally modified peptides (RiPPs) are a rapidly growing natural product superfamily with interesting and diverse bioactivities. ${ }^{1-3}$ Bottromycins (1, Scheme 1A) are RiPPs with potent activity against Gram-positive bacteria, including the problematic human pathogens MRSA and VRE. ${ }^{45}$ The bioactivity triggered several attempts at total synthesis, which was finally achieved by an inspired route requiring at least 17 steps. $^{5}$ In vitro and in vivo ${ }^{6}$ analyses of bottromycin biosynthesis have largely established the order of steps: Removal of the N-terminal methionine $^{7}$ is followed by thiazoline and macroamidine formation ${ }^{8-9}$ and removal of the follower peptide to yield $2 \mathrm{a}$ (Scheme $1 \mathrm{~B}) .^{10}$ This intermediate is then converted to $\mathbf{2} \mathbf{b}$ by the epimerase $\mathrm{Bot} H$, which results in a mixture of the two epimers (Scheme 1B). ${ }^{11}$ The D-Asp configuration of $\mathbf{2 b}$, tentatively assigned by Marfey's analysis of the 2 epimers and protein crystallography, ${ }^{11}$ is also found in the final natural product $1 . .^{5,12}$

Five-membered heterocycles that are enzymatically derived from serine, threonine or cysteine residues are frequently found in RiPPs. ${ }^{1,3}$ Their oxidation state, azoline or azole, can have a profound effect on RiPP bioactivity, ${ }^{13-14}$ and all enzymes linked with azoline oxidation in RiPP biosynthesis thus far are flavin-dependent. ${ }^{15-17}$ In contrast, it has been proposed that the oxidative decarboxylation reaction of the thiazoline in bottromycin biosynthesis is catalyzed by a P450 enzyme (BotCYP) found in the bottromycin biosynthetic gene cluster (BGC, Figure S1). ${ }^{6}$ To investigate the role of this enzyme in vitro, we used heterologously expressed and purified
BotCYP in spectral analyses to confirm an intact heme-containing protein. Incubation of BotCYP with sodium dithionite led to the observation of the typical Soret band at $448 \mathrm{~nm}$ in the spectrum with bound carbon monoxide, indicating the chemical reduction of the heme iron (Figure S2). ${ }^{18}$

Scheme 1. A Structure of bottromycin A2. B In vitro biosynthesis of $2 \mathrm{a} / \mathrm{b}$, with a proposed role for the P450 enzyme BotCYP.

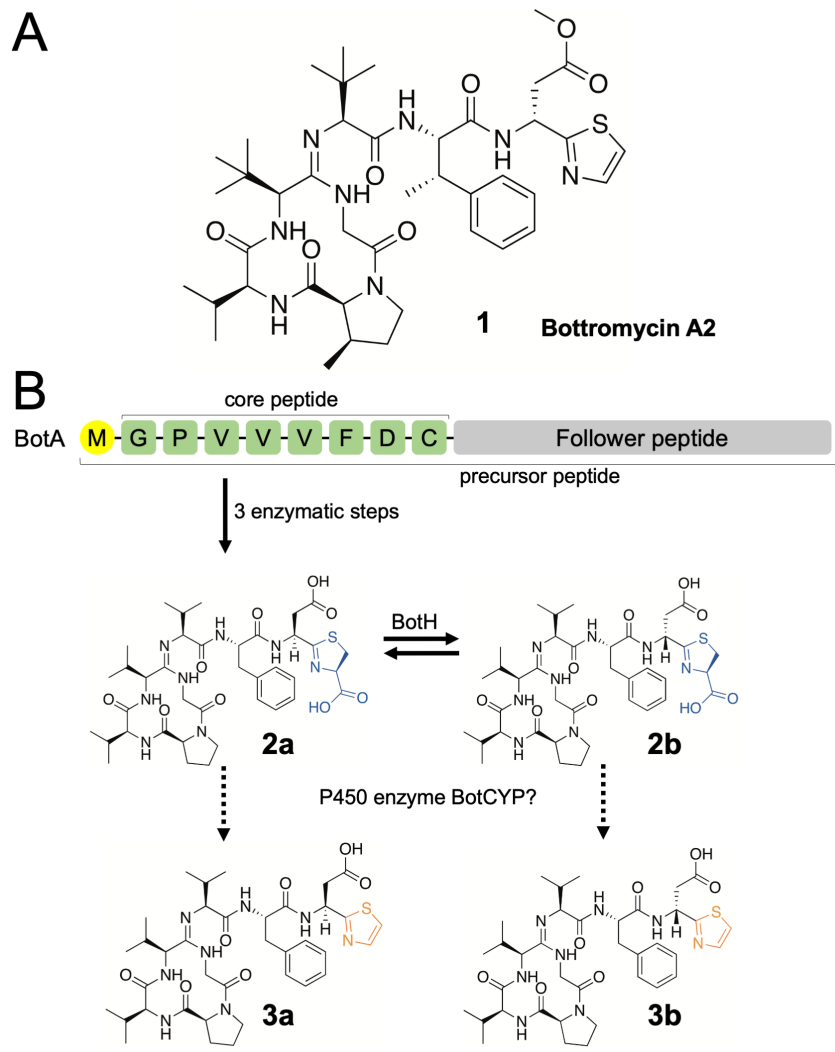

Notably, a typical reductase-ferredoxin pair required as a cofactor for cytochrome P450 enzymes is not found in the Bottromycin BGC. Enzymatic reduction of the heme iron was thus attempted via several other P450 reductase-ferredoxin pairs and the highest peak at $448 \mathrm{~nm}$ observed using the $\mathrm{P} 450$ reductase-ferredoxin pair BmCPR$\mathrm{Fdx} 2$ from Bacillus megaterium (Figure S2) ${ }^{19}$ We then produced the putative substrate mixture $\mathbf{2} \mathbf{a} / \mathbf{b}$ biochemically in vitro using three enzymes (Details can be found in the supplementary online material).-8, 10 2a/b was incubated with an optimized BotCYP : 
BmCPR : Fdx2 ratio and a molar excess of NADPH in the absence and presence of BotCYP at $30^{\circ} \mathrm{C}$ over night. The reaction mixture was subsequently analyzed by high-resolution liquid chromatography-mass spectrometry (HR-LCMS, Figures $1 \mathrm{~A}$ and $1 \mathrm{~B}$ ). In the absence of BotCYP, we observed no effect on $2 \mathrm{a} / \mathbf{b}$ $\left([\mathrm{M}+\mathrm{H}]^{+}\right.$calc.mono. $799.3807 \mathrm{Da} ;[\mathrm{M}+\mathrm{H}]^{+}{ }^{+}$obs.mono.: r.t. $3.18 \mathrm{~min}$ 799.3801 Da, error -0.75 ppm, r.t. $3.02 \mathrm{~min} 799.3796 \mathrm{Da}$, error -1.38 $\mathrm{ppm})$. In the presence of BotCYP, however, one major and one minor peak appeared, which had identical masses that corresponded to the decarboxylated, oxidized reaction product $3 \mathrm{a} / \mathrm{b}$ $\left([\mathrm{M}+\mathrm{H}]^{+}\right.$calc.mono: $753.3752 \mathrm{Da},[\mathrm{M}+\mathrm{H}]^{+}$obs.mono: r.t. $3.41 \mathrm{~min}$ 753.3743 Da, error -1.19 ppm, r.t. 3.22 min 753.3743 Da, error -1.19 ppm)) (Figure 1B). Analysis of both peaks by tandem mass spectrometry $\left(\mathrm{MS}^{2}\right)$ strongly supports the proposed structures (Figures $1 \mathrm{C}$, Tables S1 and S2).

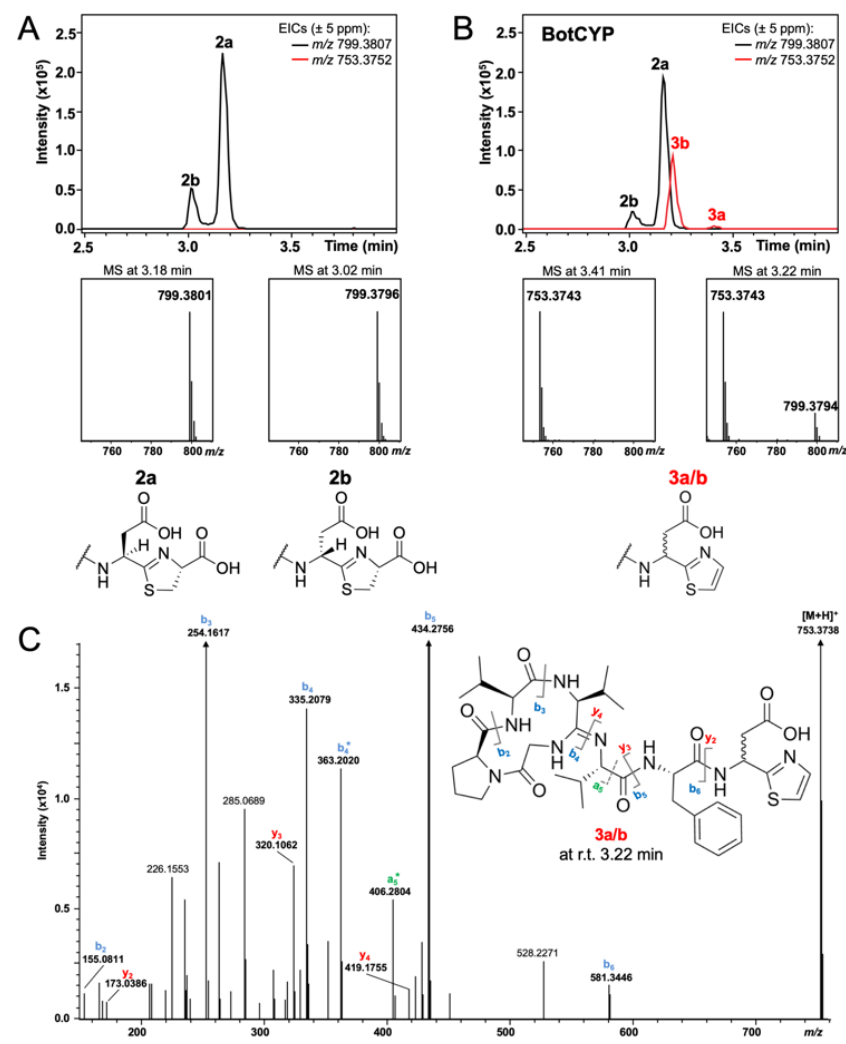

Figure 1: A Incubation of putative BotCYP substrates $\mathbf{2 a} / \mathbf{b}$ with all cofactors results in no detectable change in the HR-LCMS spectrum. $B$ Same as A, but with BotCYP added. The new species $\mathbf{3} \mathbf{a} / \mathbf{b}$ showed a loss of $46 \mathrm{Da}$, which is in agreement with the expected product. C MS ${ }^{2}$ spectrum of $3 \mathrm{a} / \mathrm{b}$. $\mathrm{a}, \mathrm{b}$ and $\mathrm{y}$ ions are indicated. The peak list and structures of the fragments $b_{2}$ and $b_{3}$ can be found in Tables $S 1$ and $S 2$.

These data establish BotCYP as sufficient for the oxidative decarboxylation of the C-terminal thiazoline to a thiazole. We estimate the yield of this reaction to be very poor $(<25 \%)$. The presence of a very small second product peak indicated that the enzyme may be stereospecific and selects one of the two available substrate epimers. Using an enzymatically produced, roughly equimolar mixture of $\mathbf{2 a}$ and $\mathbf{2} \mathbf{b}$ in time-course experiments allowed us to demonstrate that the D-Asp containing $\mathbf{2} \mathbf{b}$ peak is preferentially consumed by BotCYP (Figure 2A). To better understand BotCYP, we attempted to determine its crystal structure, but extensive screening did not yield crystals. The close homolog SalCYP from Salinispora tropica (Figure S1) was also able to perform the oxidative decarboxylation reaction on similar time-scales and with comparable yields using identical redox partners (Figures 2B and S3-4). The high-resolution (1.85 Å) structure of SalCYP was determined using single-wavelength anomalous dispersion from iron. All data collection and refinement statistics can be found in Table S3. The overall structure of SalCYP reveals a heme $b$ bound at the reactive centre, which is freely accessible via a solvent channel that leads to a large active site cavity suitable for binding very bulky substrates (Figure S5). The heme b is coordinated by Cys354, forms two salt bridges between the two heme carboxylic acid moieties and Arg104 and Arg298 side chains of SalCYP as well as extensive hydrophobic interactions (Figure S6). Surprisingly, we found the N-terminus (residues $1-20$ ) to be ordered and extended away from the protein, with the amino group of the $\mathrm{N}$-terminus coordinating the heme iron of a symmetry mate (Figure S7). To allow an unbiased analysis of the binding pocket, we engineered a new crystal contact. This mutant protein, $\mathrm{SalCYP}^{\mathrm{T}}$, retained enzymatic activity (Figure S8), readily crystallized in space group $\mathrm{P} 4_{1}$ and the structure was determined to $1.50 \AA$ resolution. The active site was now unoccupied by protein, but retained its shape and solvent channel at the entrance to the active site cavity.

A

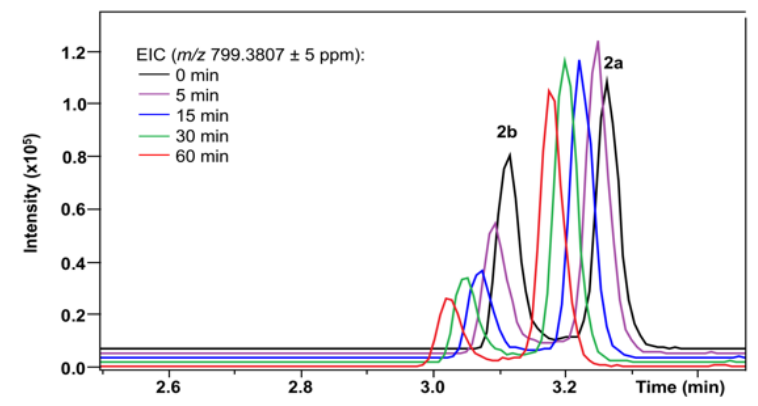

B

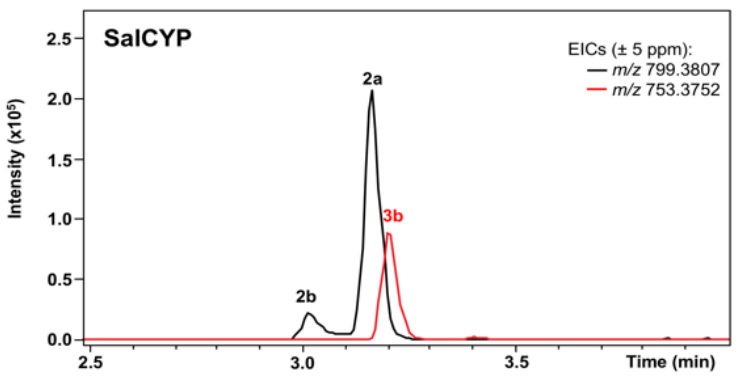

C

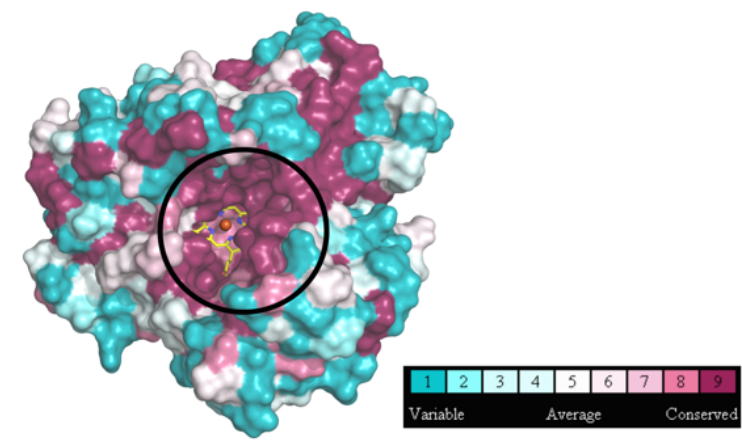

Figure 2: A HR-LCMS data show that BotCYP preferentially consumes 2b. B Analysis of a SalCYP reaction with $\mathbf{2 a} / \mathbf{b}$. One product peak is observed when SalCYP is used. C ConSurf map showing the conservation of residues around the SalCYP active site (circle) for SalCYP homologues from other bottromycin biosynthetic gene clusters (Figure 3A).

A search for structural homologs using the DALI server ${ }^{20}$ revealed the camphor hydroxylase CYP101D2 $2^{21}$ as the closest structural homolog (PDB ID 4dxy) with a $\mathrm{C}_{\alpha}$ rmsd of $2.4 \AA$ over 355 residues (Figure S9). The structural homology of SalCYP to the well- 
characterized OleTJE (PDB ID 5m0n), ${ }^{22}$ which catalyzes the oxidative decarboxylation of fatty acids, was calculated as a $\mathrm{C}_{\alpha}$ rmsd of 2.9 Å over 310 residues (Figure S9). The most striking difference between SalCYP and virtually all close structural homologs was the wide and deep active-site cleft found in SalCYP (Figure S10). The exception was the structure of TbtJ1 (PDB ID 5vws), which is the only other P450 enzyme structure from a RiPP pathway. ${ }^{23} \mathrm{Tbt} J 1 \mathrm{cat}-$ alyzes the hydroxylation of thiomuracin ${ }^{24-25}$ and possesses a similar active site topology. The residues found at the SalCYP active site are very well conserved (Figure 2C), which may reflect the need for stereocontrol for this step of bottromycin biosynthesis. Taken together, these structures thus indicate that RiPP P450 enzymes have evolved to perform selective reactions within complex linear and cyclic RiPP core peptides (Figure S10).

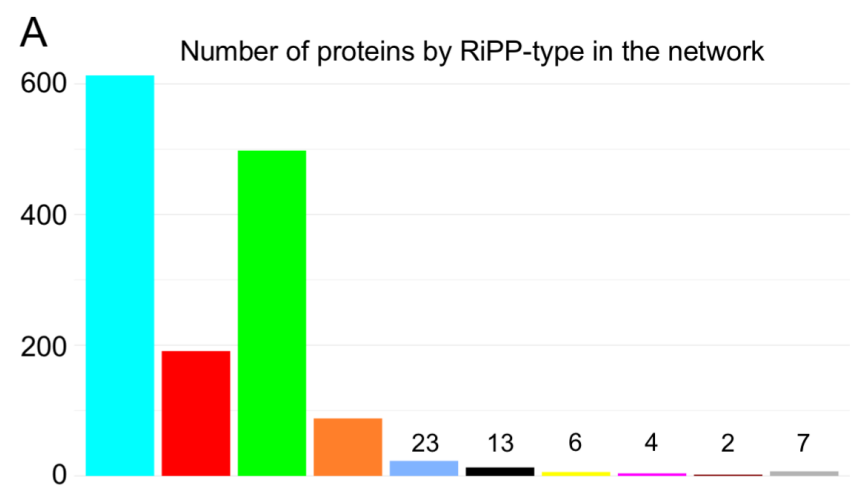

B

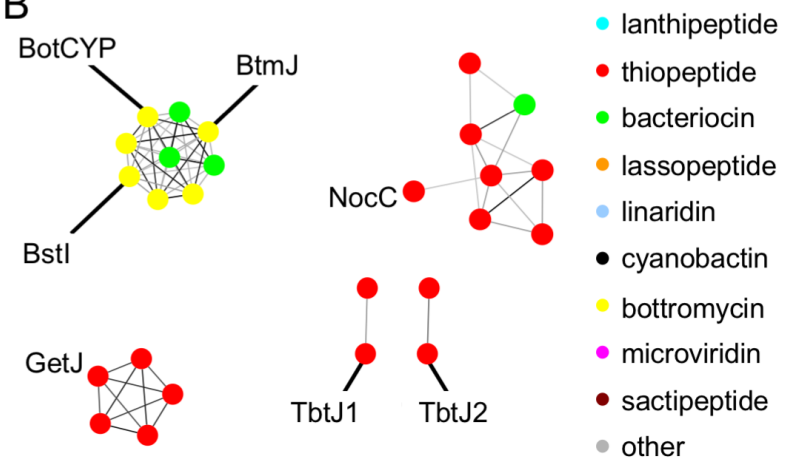

Figure 3: A Histogram displaying the number of P450 enzyme containing predicted RiPP BGCs, grouped and colored by RiPP-type (See Figure S11). B Clusters with characterized enzymes are annotated. MibO and NocV (see main text) are singlets and not displayed. Manual curation of the BotCYP-containing cluster identifies the three bacteriocin nodes as bottromycin BGCs.

We computed a sequence similarity network for BotCYP to explore the distribution of P450-like enzymes in RiPP BGCs (Figures 3 and S11). Although P450-like enzymes are widespread in bacteria (109,039 members of the Pfam family PF00067), and more than 15,000 of them are located in BGCs, only $\sim 1.5 \%(1,786)$ were found in a predicted RiPP cluster. Sequence diversity of these proteins is very large (mean sequence identity $28 \%$ ), and they are found in diverse Proteobacteria, as well as Actinobacteria, Cyanobacteria, Chloroflexi, and Bacteroidetes. BotCYP is a part of a small cluster that contains all P450 enzymes from putative bottromycin biosynthetic gene clusters, which echoes the findings for the amidohydrolase PurAH. ${ }^{10}$ We would like to note that only a handful of RiPP P450 enzymes within this network have an assigned biosynthetic function to date: MibO (microbisporicin), TbtJ1 and TbtJ2 (thiomuracin), TsrR (thiostrepton) and NocV (nocathiacin) all catalyze the hydroxylation of amino acids. ${ }^{24-27}$ GetJ from GE37468 biosynthesis catalyzes the conversion of isoleucine to $\delta$-hydroxyproline and TsrP, also involved in thiostrepton biosynthesis, catalyzes an epoxidation that triggers additional changes. ${ }^{26,28}$ None of these proteins catalyze an oxidative decarboxylation reaction, nor are they involved in heterocycle biochemistry. BotCYP thus expands the catalytic scope of P450 enzymes involved in RiPP biosynthesis, and the map of the RiPP P450 landscape we provide here may serve as a very valuable starting point to explore the functionalities of $\mathrm{P} 450$ enzymes in RiPP biosynthesis and discover novel transformations.

The spontaneous exchange between $\mathbf{2} \mathbf{a}$ and $\mathbf{2} \mathbf{b}$ is slow, ${ }^{6,11}$ and $\mathbf{2} \mathbf{b}$ (DAsp), the much less abundant epimer, was the preferred substrate for BotCYP. This led us to speculate that the poor yield of the BotCYP and SalCYP reactions may be the result of depleting $\mathbf{2 b}$. We thus added the recently characterized epimerase BotH, ${ }^{11}$ which catalyzes the rapid conversion of $\mathbf{2} \mathbf{a}$ to $\mathbf{2} \mathbf{b}$, to the reaction mixture. Analysis of reactions containing BotH and BotCYP by HR-LCMS demonstrated that the oxidative decarboxylation reaction now nearly goes to completion (Figure $4 \mathrm{~A}$ ). Since $\mathbf{2} \mathbf{b}$ contains D-Asp and was preferentially consumed by BotCYP, it may be intuitive to assume that the reaction product, $\mathbf{3 b}$, also harbors the $\mathrm{D}$-Asp found in the natural product. To probe the stereochemistry at the Asp $\mathrm{C}_{\alpha}$-position of $\mathbf{3 b}$, we enzymatically produced substrate selectively labeled with a deuteron at this position $\left(4 \mathrm{a} / \mathrm{b},[\mathrm{M}+\mathrm{H}]^{+}\right.$calc.mono: $800.3870 \mathrm{Da}$; r.t. 3.15 $\min (4 \mathrm{a})[\mathrm{M}+\mathrm{H}]^{+}$obs.mono: $800.3851 \mathrm{Da}$, error $-2.37 \mathrm{ppm}$ and r.t. $3.00 \mathrm{~min}(4 \mathrm{~b})[\mathrm{M}+\mathrm{H}]^{+}$obs.mono: $800.3852 \mathrm{Da}$, error $\left.-2.24 \mathrm{ppm}\right)$ (Figure S12).
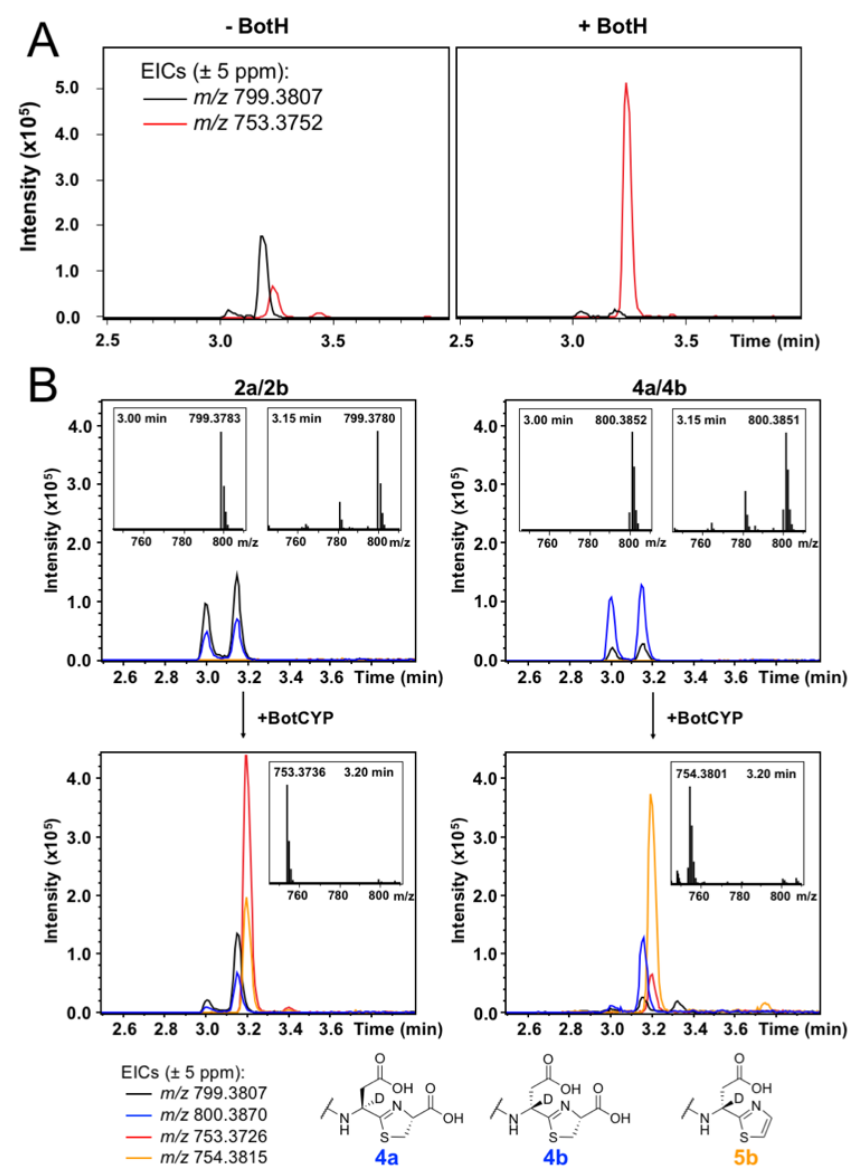

Figure 4. A Comparison of the BotCYP reaction with (right) and without (left) BotH. Addition of BotH enables virtually complete turnover. B BotCYP reaction using $4 a / b$ as a substrate. The product $5 b$ has the same retention time as $\mathbf{3 b}$, but retains its mass shift of $+1 \mathrm{Da}$. 
We then used $4 \mathbf{a} / \mathbf{b}$ as a substrate in a BotCYP reaction in $\mathrm{H}_{2} \mathrm{O}$ and the resulting peak had the same retention time and fragmentation pattern as $\mathbf{3 b}$ (Figures 4B and S13). Crucially, the product peak (5b) retained the mass shift of $+1 \mathrm{Da}\left([\mathrm{M}+\mathrm{H}]^{+}\right.$calc.mono: $754.3815 \mathrm{Da}$, $[\mathrm{M}+\mathrm{H}]^{+}$obs.mono: $754.3801 \mathrm{Da}$, error $\left.-1.86 \mathrm{ppm}\right)$, indicating that the configuration of the $\mathrm{Asp} \mathrm{C}_{\alpha}$-position is retained during the oxidative decarboxylation reaction. Presence of the deuteron at the Asp-position was confirmed by $\mathrm{MS}^{2}$ (Figure S13). Our data thus strongly imply that $\mathbf{3 b}$ represents the bottromycin core scaffold (des-methyl $\mathbf{1}$ ) harboring the D-Asp moiety found in bottromycins. Incubation of $\mathbf{5 b}$ with BotH in $\mathrm{H}_{2} \mathrm{O}$ does no longer lead to an exchange of the deuteron at the Asp $\mathrm{C}_{\alpha}$-position, indicating that heterocycle oxidation to a thiazole locks the configuration at the Asp position. ${ }^{11}$

We have demonstrated that the P450 enzyme found in the bottromycin BGC is sufficient for the oxidative decarboxylation of the Cterminal thiazoline found in the bottromycin biosynthetic intermediate $\mathbf{2} \mathbf{a} / \mathbf{b}$. The stereospecificity of BotCYP (and SalCYP) provides stereochemical resolution for the pathway, since the epimerase BotH produces a product mixture (D- and L-Asp) ${ }^{11}$ Our data also rationalize the results of a previous study attempting to produce bottromycin derivatives at the Asp-position (Asp to Thr, Ala, or Asn). ${ }^{4}$ Mutations in this position appear incompatible with BotH epimerase activity, ${ }^{11}$ resulting in very little, if any, D-amino acid substrate for BotCYP and thus no bottromycin derivative production. To function efficiently, BotCYP needs to cooperate with the epimerase BotH, ${ }^{11}$ but we were unable to detect complex formation between the two proteins (data not shown). This mirrors the results for the bottromycin biosynthetic enzymes PurCD (macrocyclase) and PurAH (amidohydrolase), which are both required for efficient macroamidine formation, but do not form a complex. ${ }^{10}$ It is still unclear if the bottromycin biosynthetic machinery is organized within the producing strains, for example by co-localizing enzymes without physical complex formation. The reconstitution of BotCYP also opens a facile, 2-pot in vitro biosynthetic route to the bottromycin core scaffold. Consequently, it might be a valuable alternative to synthetic approaches of bottromycin production, which currently still involve steps with only a moderate yield for the final macrocyclization reaction., 29 Our data may thus enable the rapid generation of bottromycin analogues for antibiotic activity testing.

\section{ASSOCIATED CONTENT}

\section{Supporting Information.}

The Supporting Information is available free of charge on the ACS Publications website.

Detailed experimental procedures and supporting information (PDF). Cytoscape session and style files (zip).

Anti-smash annotations for all BGCs including accession codes (txt). All-to-all sequence identities (gz).

\section{AUTHOR INFORMATION}

\section{Corresponding Author}

jesko.koehnke@helmholtz-hzi.de

Author contributions

S.A. and L.F. contributed equally.

\section{ORCID}

Sebastian Adam: 0000-0003-3319-6356

Laura Franz: 0000-0003-1649-3375

Jesko Koehnke: $\underline{0000-0002-7153-1365}$

\section{Funding Sources}

J.K. thanks the German Research Foundation for an Emmy Noether Fellowship (KO 4116/3-2).

\section{Notes}

No competing financial interests have been declared.

The structures of SalCYP and SalCYP ${ }^{\mathrm{T}}$ have been deposited in the protein databank (PDB IDs 6aba and 6abb, respectively).

\section{ACKNOWLEDGEMENT}

We acknowledge the use of ESRF beamlines ID29 and ID30a3. We thank Asfandyar Sikandar for providing BotH.

\section{REFERENCES}

(1) Arnison, P. G.; Bibb, M. J.; Bierbaum, G.; Bowers, A. A.; Bugni, T. S.; Bulaj, G.; Camarero, J. A.; Campopiano, D. J.; Challis, G. L.; Clardy, J.; Cotter, P. D.; Craik, D. J.; Dawson, M.; Dittmann, E.; Donadio, S.; Dorrestein, P. C.; Entian, K. D.; Fischbach, M. A.; Garavelli, J. S.; Goransson, U.; Gruber, C. W.; Haft, D. H.; Hemscheidt, T. K.; Hertweck, C.; Hill, C.; Horswill, A. R.; Jaspars, M.; Kelly, W. L.; Klinman, J. P.; Kuipers, O. P.; Link, A. J.; Liu, W.; Marahiel, M. A.; Mitchell, D. A.; Moll, G. N.; Moore, B. S.; Muller, R.; Nair, S. K.; Nes, I. F.; Norris, G. E.; Olivera, B. M.; Onaka, H.; Patchett, M. L.; Piel, J.; Reaney, M. J.; Rebuffat, S.; Ross, R. P.; Sahl, H. G.; Schmidt, E. W.; Selsted, M. E.; Severinov, K.; Shen, B.; Sivonen, K.; Smith, L.; Stein, T.; Sussmuth, R. D.; Tagg, J. R.; Tang, G. L.; Truman, A. W.; Vederas, J. C.; Walsh, C. T.; Walton, J. D.; Wenzel, S. C.; Willey, J. M.; van der Donk, W. A., Ribosomally Synthesized and PostTranslationally Modified Peptide Natural Products: Overview and Recommendations for a Universal Nomenclature. Nat Prod Rep 2013, 30, 108-60.

(2) Ortega, M. A.; van der Donk, W. A., New Insights into the Biosynthetic Logic of Ribosomally Synthesized and PostTranslationally Modified Peptide Natural Products. Cell Chem Biol 2016, 23, 31-44.

(3) Montalban-Lopez, M.; Scott, T. A.; Ramesh, S.; Rahman, I. R.; van Heel, A. J.; Viel, J. H.; Bandarian, V.; Dittmann, E.; Genilloud, O.; Goto, Y.; Grande Burgos, M. J.; Hill, C.; Kim, S.; Koehnke, J.; Latham, J. A.; Link, A. J.; Martinez, B.; Nair, S. K.; Nicolet, Y.; Rebuffat, S.; Sahl, H. G.; Sareen, D.; Schmidt, E. W.; Schmitt, L.; Severinov, K.; Sussmuth, R. D.; Truman, A. W.; Wang, H.; Weng, J. K.; van Wezel, G. P.; Zhang, Q.; Zhong, J.; Piel, J.; Mitchell, D. A.; Kuipers, O. P.; van der Donk, W. A., New Developments in Ripp Discovery, Enzymology and Engineering. Nat Prod Rep 2020.

(4) Hou, Y.; Tianero, M. D.; Kwan, J. C.; Wyche, T. P.; Michel, C. R.; Ellis, G. A.; Vazquez-Rivera, E.; Braun, D. R.; Rose, W. E.; Schmidt, E. W.; Bugni, T. S., Structure and Biosynthesis of the Antibiotic Bottromycin D. Org Lett 2012, 14, 5050-3.

(5) Shimamura, H.; Gouda, H.; Nagai, K.; Hirose, T.; Ichioka, M.; Furuya, Y.; Kobayashi, Y.; Hirono, S.; Sunazuka, T.; Omura, S., Structure Determination and Total Synthesis of Bottromycin A2: A Potent Antibiotic against Mrsa and Vre. Angew Chem Int Ed Engl 2009, 48, 914-7.

(6) Crone, W. J.; Vior, N. M.; Santos-Aberturas, J.; Schmitz, L. G.; Leeper, F. J.; Truman, A. W., Dissecting Bottromycin Biosynthesis Using Comparative Untargeted Metabolomics. Angew Chem Int Ed Engl 2016, 55, 9639-43.

(7) Mann, G.; Huo, L.; Adam, S.; Nardone, B.; Vendome, J.; Westwood, N. J.; Muller, R.; Koehnke, J., Structure and Substrate Recognition of the Bottromycin Maturation Enzyme Botp. Chembiochem 2016, 17, 2286-2292.

(8) Franz, L.; Adam, S.; Santos-Aberturas, J.; Truman, A. W.; Koehnke, J., Macroamidine Formation in Bottromycins Is Catalyzed by a Divergent Ycao Enzyme. J Am Chem Soc 2017, 139, 18158-18161. 
(9) Schwalen, C. J.; Hudson, G. A.; Kosol, S.; Mahanta, N.; Challis, G. L.; Mitchell, D. A., In Vitro Biosynthetic Studies of Bottromycin Expand the Enzymatic Capabilities of the Ycao Superfamily. J Am Chem Soc 2017, 139, 18154-18157.

(10) Sikandar, A.; Franz, L.; Melse, O.; Antes, I.; Koehnke, J., Thiazoline-Specific Amidohydrolase Purah Is the Gatekeeper of Bottromycin Biosynthesis. J Am Chem Soc 2019, 141, 9748-9752. (11) Sikandar, A.; Franz, L.; Adam, S.; Santos-Aberturas, J.; Horbal, L.; Luzhetskyy, A.; Truman, A. W.; Kalinina, O. V.; Koehnke, J., The Bottromycin Epimerase Both Defines a Group of Atypical Alpha/Beta-Hydrolase-Fold Enzymes. Nat Chem Biol 2020, 16, 1013-1018.

(12) Gouda, H.; Kobayashi, Y.; Yamada, T.; Ideguchi, T.; Sugawara, A.; Hirose, T.; Omura, S.; Sunazuka, T.; Hirono, S., Three-Dimensional Solution Structure of Bottromycin A2: A Potent Antibiotic Active against Methicillin-Resistant Staphylococcus Aureus and Vancomycin-Resistant Enterococci. Chem Pharm Bull (Tokyo) 2012, 60, 169-71.

(13) Gao, S.; Ge, Y.; Bent, A. F.; Schwarz-Linek, U.; Naismith, J. H., Oxidation of the Cyanobactin Precursor Peptide Is Independent of the Leader Peptide and Operates in a Defined Order. Biochemistry 2018, 57, 5996-6002.

(14) Houssen, W. E.; Jaspars, M., Azole-Based Cyclic Peptides from the Sea Squirt Lissoclinum Patella: Old Scaffolds, New Avenues. Chembiochem 2010, 11, 1803-15.

(15) Bent, A. F.; Mann, G.; Houssen, W. E.; Mykhaylyk, V.; Duman, R.; Thomas, L.; Jaspars, M.; Wagner, A.; Naismith, J. H., Structure of the Cyanobactin Oxidase Thcox from Cyanothece Sp. Pcc 7425, the First Structure to Be Solved at Diamond Light Source Beamline 123 by Means of S-Sad. Acta Crystallogr D Struct Biol 2016, 72, 1174-1180.

(16) Li, Y. M.; Milne, J. C.; Madison, L. L.; Kolter, R.; Walsh, C. T., From Peptide Precursors to Oxazole and ThiazoleContaining Peptide Antibiotics: Microcin B17 Synthase. Science 1996, 274, 1188-93.

(17) Melby, J. O.; Li, X.; Mitchell, D. A., Orchestration of Enzymatic Processing by Thiazole/Oxazole-Modified Microcin Dehydrogenases. Biochemistry 2014, 53, 413-22.

(18) Guengerich, F. P.; Martin, M. V.; Sohl, C. D.; Cheng, Q., Measurement of Cytochrome P450 and Nadph-Cytochrome P450 Reductase. Nat Protoc 2009, 4, 1245-51.

(19) Milhim, M.; Gerber, A.; Neunzig, J.; Hannemann, F.; Bernhardt, R., A Novel Nadph-Dependent Flavoprotein Reductase from Bacillus Megaterium Acts as an Efficient Cytochrome P450 Reductase. J Biotechnol 2016, 231, 83-94.

(20) Holm, L.; Laakso, L. M., Dali Server Update. Nucleic Acids Res 2016, 44, W351-5.

(21) Bell, S. G.; Yang, W.; Dale, A.; Zhou, W.; Wong, L. L., Improving the Affinity and Activity of Cyp101d 2 for Hydrophobic Substrates. Appl Microbiol Biotechnol 2013, 97, 3979-90.

(22) Wang, J. B.; Lonsdale, R.; Reetz, M. T., Exploring Substrate Scope and Stereoselectivity of P450 Peroxygenase Oletje in Olefin-Forming Oxidative Decarboxylation. Chem Commun (Camb) 2016, 52, 8131-3.

(23) Gober, J. G.; Ghodge, S. V.; Bogart, J. W.; Wever, W. J.; Watkins, R. R.; Brustad, E. M.; Bowers, A. A., P450-Mediated Non-Natural Cyclopropanation of Dehydroalanine-Containing Thiopeptides. ACS Chem Biol 2017, 12, 1726-1731.

(24) Hudson, G. A.; Zhang, Z.; Tietz, J. I.; Mitchell, D. A.; van der Donk, W. A., In Vitro Biosynthesis of the Core Scaffold of the Thiopeptide Thiomuracin. J Am Chem Soc 2015, 137, 160125.

(25) Zhang, Z.; Hudson, G. A.; Mahanta, N.; Tietz, J. I.; van der Donk, W. A.; Mitchell, D. A., Biosynthetic Timing and Substrate Specificity for the Thiopeptide Thiomuracin. J Am Chem Soc 2016, 138, 15511-15514.

(26) Zheng, Q.; Wang, S.; Liao, R.; Liu, W., PrecursorDirected Mutational Biosynthesis Facilitates the Functional
Assignment of Two Cytochromes P450 in Thiostrepton Biosynthesis. ACS Chem Biol 2016, 11, 2673-2678.

(27) Bai, X.; Guo, H.; Chen, D.; Yang, Q.; Tao, J.; Liu, W., Isolation and Structure Determination of Two New NosiheptideType Compounds Provide Insights into the Function of the Cytochrome P450 Oxygenase Nocv in Nocathiacin Biosynthesis. Organic Chemistry Frontiers 2020, 7, 584-589.

(28) Young, T. S.; Walsh, C. T., Identification of the Thiazolyl Peptide Ge37468 Gene Cluster from Streptomyces Atcc 55365 and Heterologous Expression in Streptomyces Lividans. Proc Natl Acad Sci U S A 2011, 108, 13053-8.

(29) Yamada, T.; Yagita, M.; Kobayashi, Y.; Sennari, G.; Shimamura, H.; Matsui, H.; Horimatsu, Y.; Hanaki, H.; Hirose, T.; S, O. M.; Sunazuka, T., Synthesis and Evaluation of Antibacterial Activity of Bottromycins. J Org Chem 2018, 83, 7135-7149. 